\title{
SARCOPENIA VERSUS CAQUEXIA
}

\author{
M ónica B ermúdez M D*, Rodrigo Becerra M D**, J uan Carlos Galvis M D***
}

\section{Resumen}

Las enfermedades crónicas y la edad se asocian con deterioro del estado nutricional, pérdida de masa, fuerza muscular, alteración en la calidad de vida y aumento en morbilidad y mortalidad. Se revisan la definición y los criterios para diferenciar caquexia de sarcopenia. Es importante investigar los factores asociados con pérdida de las reservas grasas y de tejido muscular en las dos situaciones, para desarrollar estrategias terapéuticas y preventivas en adultos sanos y con patologías. El consenso europeo (2010) sobre las alteraciones de la masa muscular determinó los criterios para diferenciar caquexia y sarcopenia. La primera se consideró complicación tardía e inevitable de distintas patologías crónicas. Recientes evidencias clínicas y experimentales indican que los mecanismos involucrados en su aparición operan en forma temprana y sugieren intervenciones adecuadas que podrían prevenir o demorar la aparición de este síndrome.

Palabras clave: caquexia, sarcopenia, enfermedades crónicas, edad.

\section{SARCOPENIA VERSUS CACHEXIA}

\section{Abstract}

Chronic diseases and aging are associated with deterioration of nutritional status, loss of muscle mass and function, impaired quality of life and increased risk for morbidity and mortality. This paper reports the definition of cachexia and sarcopenia, as well as, the criteria for the differentiation between them. Investigating the factors associated to the loss of fat and muscle tissue in both conditions to develop therapeutic and preventive strategies in healthy and ill adults, is very important. The 2010 European consensus on muscle mass alterations determined the differentiation criteria for cachexia and sarcopenia. Cachexia has been considered a late and ineluctable event complicating many chronic diseases. Recent clinical and experimental evidences indicate that mechanisms involved are operating early during the disease, suggesting that appropriate interventions might be effective in preventing or delaying the onset of this syndrome.

Key words: cachexia, sarcopenia, chronic diseases, age.

Fecha recibido: octubre I4 de 2014 - Fecha aceptado: noviembre 27 de 2014

* Especialista en Medicina de la Actividad Física y del Deporte. Fundación Universitaria de Ciencias de la Salud. Bogotá DC. Colombia

** Especialista en Medicina de la Actividad Física y del Deporte. Instructor Asistente. Fundación Universitaria de Ciencias de la Salud. Servicio de Rehabilitación Hospital Infantil Universitario de San José. Grupo de Investigación en Actividad Física GIAF - FUCS. Bogotá DC. Colombia.
*** Especialista en Medicina del Deporte. Instructor Asistente, Fundación Universitaria de Ciencias de la Salud. Jefe del Servicio de Rehabilitación del Hospital Infantil Universitario de San José.

Unidad Médica de Actividad Física y Deporte UMAF-UMFIDE de la IPS del Deporte. Grupo de Investigación en Actividad Física GIAF-FUCS. Bogotá DC. Colombia. 


\section{Introducción}

El envejecimiento es un fenómeno biológico complejo de gran importancia. ${ }^{1}$ Todos los cambios con la edad tienen bases celulares. Es un proceso estudiado a nivel celular bajo condiciones ambientales definidas y controladas. ${ }^{2}$ En los últimos años las enfermedades y discapacidades relacionadas con la edad han Ilegado a ser de gran interés e importancia para la salud. Esto sucede en especial en el mundo occidental, donde la mejora en la salud, el cambio del estilo de vida y la higiene han reducido las principales causas de muerte prevalentes en otras épocas como las enfermedades infecciosas, por lo que la esperanza de vida media ha aumentado de manera sorprendente. La pérdida de peso es el principal factor que incrementa la mortalidad en la población geriátrica. La conexión entre el apetito y la buena salud se ha conocido desde hace cientos de años. A demás la pérdi da brusca del apetito en los ancianos se asocia con su próximo fallecimiento. El tratamiento de la malnutrición y la pérdida de peso puede ayudar a mejorar diferentes condiciones médicas. $^{2}$

Se ha demostrado que el soporte nutricional acorta el tiempo de rehabilitación después de fracturas de cadera. En pacientes geriátricos hospital izados la presencia de concentraciones bajas de al búmina en suero acompañadas de pérdida de peso aumentan el riesgo de muerte. $L$ a reducción del peso obedece a distintas causas. ${ }^{3}$ En la vejez conduce a caquexia con una pérdida preferencial de masa magra respecto a tejido adiposo. En la senectud hay un aumento del gasto energético basal que podría ser una de las causas de la pérdida de peso. El desgaste y la caquexia están asociados con severas consecuencias fisiológicas, psicológicas e inmunológicas ${ }^{4}$, dando lugar a un aumento del número de infecciones, úlceras de decúbito y al gunas muertes. L a baja involuntaria de peso en la vejez fue de $13 \%$ en un grupo de 247 varones residentes en centros de tercera edad de 65 años 0 más; cuando es más de $4 \%$ del peso corporal constituye un buen elemento para predecir una elevada tasa de mortal idad. La malnutrición puede causar desórdenes cognitivos y del estado de ánimo. ${ }^{5}$ En la vejez los desórdenes médicos, cognitivos y psiquiátricos pueden disminuir la capacidad para la realización autónoma de las actividades de la vida diaria, reduciendo así la calidad de vida y aumentando la frecuencia de procesos secundarios, hospitalizaciones y la necesidad de cuidados especiales. ${ }^{6,7}$ Por 10 tanto, el peso y la nutrición adecuados son necesarios para una buena calidad de vida y para una salud óptima en centros de la tercera edad. ${ }^{8}$

La estrecha relación entre las enfermedades crónicas y el deterioro del estado nutricional afecta la calidad de vida e incrementa el riesgo para la mortalidad y la morbilidad. En el siglo III A.C. Hipócrates describió el síndrome de pérdida asociado con la enfermedad terminal. ${ }^{9}$ El espectro de las anormal idades metabólicas y nutricionales secundarias a enfermedades crónicas es un tema amplio y de origen multifactorial. En 2005 el Special Interest G roup (SIG) sobre caquexia y anorexia en enfermedades crónicas, enfocó sus objetivos principales sobre el desarrollo y propagación del conocimiento sobre los aspectos básicos y clínicos de estas patologías, su prevención y tratamiento. La falta de una definición simple y aceptada para la caquexia sigue representado un problema que conduce a errores clínicos relevantes, a la falta o atraso en la identificación, la prevención inadecuada y con frecuencia a tratamientos ineficaces. La sarcopenia es un concepto que describe la pérdida de masa y fuerza musculares asociada con la edad, y en fases avanzadas puede causar discapacidad y dependencia. El conocimiento cada vez mayor de la bioquímica y los procesos moleculares subyacentes hace posible que hoy día se propongan nuevos tratamientos que podrían revertir la disminución corporal. ${ }^{10}$

\section{¿Cómo se define sarcopenia?}

El termino se deriva del griego sarx (carne) y penia (pobre). ${ }^{11}$ Es una condición caracterizada por la pérdida de masa y fuerza musculares. ${ }^{11,12} \mathrm{EI}$ músculo representa el $60 \%$ de la reserva proteica. Su disminución es responsable directa de la alteración funcional con pérdida de fuerza, aumentando la probabilidad de caídas y pérdida de autonomía. ${ }^{13,14} \mathrm{~L}$ a función respiratoria se ve afectada con una capacidad vital reducida. A unque la sarcopenia se considera una afección del envejecimiento ${ }^{15}$, su desarrollo puede asociarse con otras condiciones que se observan en personas mayores 
(Figura 1). A I igual que la osteopenia, puede verse en jóvenes con enfermedades inflamatorias, endocrinas o neurológicas y se relaciona con la disminución de su funcionalidad. ${ }^{16}$

El European Working Group on Sarcopenia in Older People (EWGSOP) ${ }^{16}$, propuso considerar la sarcopenia como primaria cuando la edad solo explica la pérdida de masa y función musculares o secundaria cuando está relacionada con una o más causas. ${ }^{10}$

\section{Sarcopenia y edad}

Es la pérdida de la masa y fuerza musculares asociada con la edad. ${ }^{17-20} \mathrm{~L}$ as características son reducción de la masa muscular y el área transversal de las fibras, infiltración del músculo por grasa y tejido conectivo, disminución del tamaño y número de fibras musculares tipo 1 y $2{ }^{21}$, acumulación de núcleos internos, desarreglo de los miofilamentos y líneas Z, proliferación del retículo sarcoplásmico y del sistema de los túbulos $T$ y menor número de unidades motoras. ${ }^{22}$ L a fisiopatología de la sarcopenia en los ancianos es compleja. Existe una multitud de procesos internos y externos que contribuyen a su desarrollo. Con respecto a los primeros las influencias más importantes son la reducción de hormonas anabólicas como testosterona, estrógenos, hormona del crecimiento, factor de

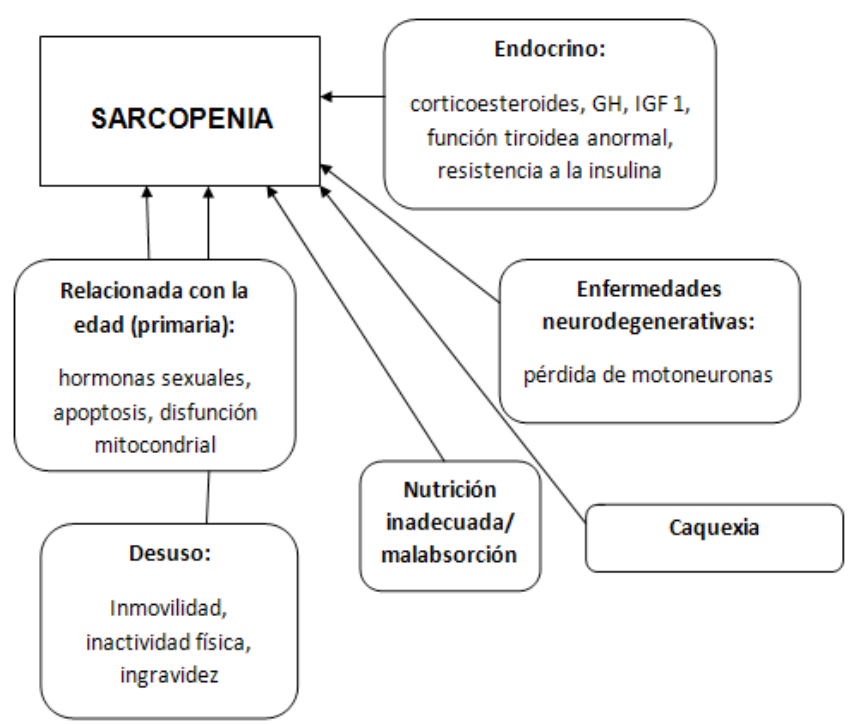

Figura I.Mecanismos de la sarcopenia.Tomado de Cruz-Jentoft, Sarcopenia: consenso europeo sobre su definición y diagnóstico.Age and Ageing 20 I0; 39:4 | 2-423. I crecimiento similar a la insulina IGF-1 ${ }^{23}$, incremento de la actividad apoptótica en las miofibrillas, aumento de citocinas proinflamatorias (en especial factor de necrosis tumoral TNF-, IL-6), el estrés oxidativo, los cambios de la función mitocondrial de las células musculares y una disminución en el número de motoneuronas alfa. Entre las influencias externas encontramos la ingesta deficiente de energía y proteínas que contribuyen a la pérdida y función musculares. EI poco aporte de vitamina $D$ se asocia con baja funcionalidad en las personas mayores así como las comorbilidades agudas y crónicas que también contribuyen al desarrollo de la sarcopenia. E stas reducen la actividad física y aumentan los periodos de reposo en cama y por otra parte aumentan la expresión de citocinas proinflamatorias que desencadenan proteólisis. ${ }^{1,23}$

\section{¿Cómo diagnosticar la sarcopenia?}

El diagnóstico se basa en la disminución del porcentaje de masa muscular, cuando es mayor o igual a dos desviaciones estándar por debajo de la media en adultos jóvenes del mismo género y etnia. A I igual que para la osteopenia y la osteoporosis, se hacen necesarios los valores de referencia en diversas poblaciones basados en la raza, edad, género y localización, lo cual ayuda a precisar con mayor exactitud el diagnóstico. La presencia y combinación de los dos criterios siguientes asegura o incrementa el grado de enfermedad: 1) reducción de la fuerza muscular, medida con el gesto de aprensión de la mano con valores inferiores a $30 \mathrm{k}$ para hombres y $20 \mathrm{k}$ para mujeres y 2) baja velocidad de la marcha, por ejemplo, al caminar por debajo de $0.8 \mathrm{~m} / \mathrm{s}$ en la prueba de caminar $4 m^{1,24}$

\section{¿Cómo definir la caquexia?}

El término se deriva del griego kakos (malo) y héxis (condición). En la actualidad la definición consensuada señala que es "un síndrome metabólico complejo asociado con una enfermedad subyacente caracterizada por la pérdida de múscul o con o sin masa grasa. Su característica prominente es la reducción del peso en adultos (corregido por retención de líquidos) o la fal ta de crecimiento en niños (excluyendo causas endocrinas). 
Esta enfermedad de desgaste como norma se asocia con anorexia, inflamación, resistencia a la insulina y aumento en la degradación de proteínas musculares. El desgaste es diferente del ocurrido por inanición, sarcopenia, lipoatrofia, así como del producido por depresión primaria, malabsorción o hipertiroidismo y se asocia con un aumento de la morbilidad".

En la clínica la caquexia es relevante ya que aumenta la morbilidad y la mortalidad de los pacientes. Los factores que contribuyen a su aparición son anorexia y cambios metabólicos, es decir incremento del estado metabólico y de la proteólisis muscular, así como alteración en el metabolismo de carbohidratos, proteínas y lípidos. Cachexia Society ha puesto hace poco a disposición una definición clínica ${ }^{1,25}$ cuyo criterio diagnóstico fundamental es la pérdida de peso (por lo menos $5 \%$ del corporal durante los últimos diez a doce meses) 0 bien cuando el índice de masa corporal (IM C) es menor de $20 \mathrm{k} / \mathrm{m}^{2.26}$, además de la presencia de más de tres de las siguientes cinco características: disminución de la fuerza muscular, fatiga, anorexia, bajo índice de masa libre grasa y alteraciones bioquímicas (aumento de marcadores inflamatorios PCR e IL-6, anemia con hemoglobina $<12 \mathrm{~g} / \mathrm{dL}$ e hipoal buminemia $<3.2 \mathrm{~g} / \mathrm{dL}$ ). ${ }^{26}$ Otros autores refieren que la caquexia se ha reconocido como un síndrome asociado con muchas enfermedades, sin embargo el conocimiento sobre los mecanismos subyacentes que la producen es aún incipiente. ${ }^{25,26} \mathrm{El}$ hecho de que no existiera una definición precisa de este síndrome sino hasta 2006 hizo que durante muchos años su diagnóstico y tratamiento sufrieran un retraso. ${ }^{27}$

L a caquexia se clasifica como leve, moderada o grave de acuerdo con la pérdida de peso de 5\%, 10\% o 15\% en los doce meses previos. A unque en la práctica clínica resul ta difícil contar con los recursos para evaluarla en forma correcta, la pérdida de peso es indicativa y de hecho se considera un indicador predictivo de morbilidad y mortalidad en estos casos. ${ }^{25} \mathrm{~L}$ a caquexia es poco reconocida y menos aún tratada.

En el proceso existe aumento de la excreción de nitrógeno y en teoría mayor síntesis de proteínas por la gran disponibilidad de aminoácidos resul tantes de la degra- dación acelerada de proteína muscular. ${ }^{28} \mathrm{~L}$ a pérdida de tejido adiposo se relaciona con malignidad ${ }^{29}$, falla cardiaca crónica ${ }^{30}$, infección por $\mathrm{VIH}^{31}$ y enfermedad renal crónica. ${ }^{32}$

L a caquexia implica un proceso importante de deterioro físico en comparación con el cuadro de desnutrición convencional, debido a la pérdida acelerada del músculo esquelético con relación al tejido adiposo, a la presencia de citocinas proinflamatorias y al incremento en la síntesis de proteínas de fase aguda, lo que contribuye al al to gasto energético y la pérdida de peso. ${ }^{33,34}$ E sta última es la característica más importante en adultos. La nueva definición fue acordada por la E uropean Society of Clinical Nutrition and M etabolism Special Interest Groups (ESPE N SIG) ${ }^{1}$ y la complementaron estableciendo estadios para que el diagnóstico en su fase inicial permitiera identificar temprano los marcadores y mejorar las medidas preventivas. En el ámbito clínico la posibilidad de identificar personas mayores en una etapa temprana de caquexia puede dar lugar a aplicaciones innovadoras. ${ }^{10} \mathrm{~L}$ a inflamación provoca mayor degradación de las proteínas musculares y resistencia a la insulina, que son las causas principales y comunes. El tratamiento podría mejorar la sarcopenia relacionada con la caquexia, mientras que otros enfoques como la actividad física solo tienen impacto en la sarcopenia. ${ }^{10}$

Es más difícil diagnosticar caquexia porque puede ser un proceso latente hasta que resulta aparente en la clínica, pero se caracteriza por el aumento de actividad de las rutas proteolíticas intracelulares. ${ }^{35} \mathrm{~L}$ os pacientes tienen muy bajo peso, pérdida importante de masa grasa y reducción de la muscular, aunque con proteínas séricas normales, mientras que en la anorexia franca el metabolismo es casi normal y la desnutrición puede corregirse con suplementación (alimentación enteral o parenteral). El éxito terapéutico en la caquexia por cáncer con frecuencia está limitado por las al teraciones metabólicas presentes. ${ }^{36}$

\section{¿La inflamación interviene en la patogénesis de la caquexia?}

L a inflamación sistémica es una característica común de las enfermedades crónicas. Juega papel funda- 
mental en la patogénesis de la caquexia y permite la identificación de ésta. En la actualidad se cree que un desequilibrio entre factores proinflamatorios (TNF- , IL-1, IL -6, IFN-gama) y antiinflamatorios (citocinas IL -4, IL -12, IL-15), contribuyen a la caquexia. Por otro lado, niveles elevados de IL - 6 se correlacionan con valores al tos de proteína $C$ reactiva (PCR) y concomitante con la pérdida de peso corporal. ${ }^{1}$

Tal vez la asociación de síntomas más clara es el conjunto de anorexia y caquexia que terminan por formar un verdadero círculo vicioso, con mayor deterioro nutricional y mayor fatigabilidad. Se usa la denominación síndrome anorexia/caquexia en pacientes con cáncer, el término wasting syndrome se ha utilizado en pacientes con SIDA y el de sarcopenia para la pérdida de masa muscular de los ancianos. Pese a su origen distinto, comparten mecanismos comunes, incluyendo el pertenecer a un mismo escenario de cambios inflamatorios. L a presencia de este síndrome entraña un peor pronóstico, como se puede comprobar en pacientes con cáncer 0 insuficiencia cardiaca con 0 sin caquexia. ${ }^{37}$ Pese al que es más bien fácil de reconocer, uno de los primeros problemas al enfrentar esta situación clínica es que no existe una definición estándar. Davis y Dickerson lo definieron como: "baja de más de un $10 \%$ del peso premórbido, asociado con pérdida de proteína visceral y muscular y a lipolisis". ${ }^{38}$ K otler lo describe como "pérdida acelerada de músculo esquelético en el contexto de una respuesta inflamatoria crónica".39,40 En la Tabla 1 se resumen criterios diagnósticos que se proponen como una definición operativa para el diagnóstico de caquexia.

\begin{tabular}{l}
\multicolumn{1}{|c|}{ Tabla I. Definición de caquexia* } \\
\hline \multicolumn{1}{|c|}{ Pérdida de peso } \\
\hline $\begin{array}{l}\text { >5\% comparado con el peso estable premórbido } \\
>5 \% \text { durante los } 6 \text { meses previos } \\
>2 \% \text { dentro del período de I mes } \\
>2-3 \text { k durante los últimos } 2 \text { meses } \\
\text { Ingesta de alimentos por vía oral < calorias/k al día y pérdida de peso } \\
\text { Disminución de II k en los } 2 \text { meses previos }\end{array}$ \\
\hline \multicolumn{1}{|c|}{ Síntomas asociados } \\
\hline $\begin{array}{l}\text { Anorexia } \\
\text { Reducción de ingesta calórica } \\
\text { Astenia } \\
\text { Nausea crónica } \\
\text { Pérdida de músculo y tejido graso } \\
\text { Bajas concentraciones de albúmina sérica } \\
\text { Edema periférico }\end{array}$ \\
\hline
\end{tabular}

Adaptado de Cereceda G Luis. Mecanismos de la Caquexia. Rev Med Clin Condes 2007;18(4):349-55.40
En la actualidad se considera un fenómeno complejo, de hecho es posible ver el desarrollo del cuadro de caquexia/anorexia aún en pacientes con tumores pequeños $(0,01 \%$ del peso corporal), constituyendo un verdadero cuadro paraneoplásico. Es frecuente que en estos pacientes la situación se vea agravada por factores transitorios que colaboran a disminuir la ingesta (dolor bucofaríngeo, infecciones agudas, disfagia, xerostomía, medicamentos) En los casos en que predomina un déficit de ingesta, el cuadro de desnutrición que se desarrolla muestra una disminución del gasto energético con conservación inicial de las proteínas musculares viscerales y uso de cuerpos cetónicos como fuente de energía, resultando en una pérdida de peso con predominio de la grasa corporal. Esto es conocido como caquexia secundaria que en inglés se denomina starvation y equivale casi a inanición. El fenómeno de caquexia se desarrolla desde el inicio y se acompaña de un aumento de la tasa metabólica basal, con movilización de proteínas y lípidos, resultando en una pérdida más acelerada de peso a expensas de los compartimientos proteícos y grasos. ${ }^{39}$

La sarcopenia/caquexia del anciano es un fenómeno multifactorial que incluye anorexia y disminución del gasto energético basal. Hay elevación de citoquinas y actividad inflamatoria. Es también frecuente el hipogonadismo, siendo la disminución de testosterona un factor que el eva la leptina, que a su vez conduce a más anorexia. A demás los mecanismos reparativos del músculo están deficientes. ${ }^{39}$

\section{¿Hay diferencia entre caquexia y malnutrición?}

La primera se considera como el resultado de una compleja interacción entre la afección de base, la enfermedad relacionada con alteraciones metabólicas y en al gunos casos, la reducida disponibilidad de nutrientes debida a baja ingesta, alteración en la absorción y aumento de las pérdidas, o bien una combinación de estos. La malnutrición es un estado de nutrición en el cual una deficiencia o exceso de energía, proteínas y otros nutrientes causan efectos adversos sobre tejidos, forma corporal, función y respuesta clínica. La disponibilidad reducida de los nutrientes puede ser 
un componente que juega un papel en la patogénesis de la caquexia. En efecto, cabe señalar que si bien no siempre los desnutridos son caquécticos, es invariable que todos los caquécticos, sean desnutridos. ${ }^{1}$

\section{¿La caquexia se asocia con cambios en la composición corporal?}

Una de las características de la caquexia es la reducción del peso corporal que se produce por la pérdida de grasa y masa magra. En la forma avanzada la retención de agua puede ocurrir como consecuencia de hipoal buminemia severa, que puede explicar el aumento en el peso a pesar de la severa pérdida corporal. Lo mismo puede ocurrir en pacientes con insuficiencia cardiaca grave, cirrosis hepática o falla renal, donde la pérdida de peso puede estar enmascarada por la retención de líquido. $L$ a reducción de la masa muscular esquelética se considera como la característica fenotípica clínica relevante de la caquexia, independiente de la enfermedad subyacente, por lo tanto debe distinguirse de otras formas de pérdida muscular, en particular de la sarcopenia relacionada con la edad. ${ }^{1}$

\section{¿Es posible diferenciar caquexia de otras condiciones asociadas con sarcopenia?}

L a pérdida de masa muscular es una característica de la caquexia, mientras que la mayoría de los sujetos sarcopénicos no son caquécticos. L as personas sin pérdida de peso, anorexia ni respuesta inflamatoria sistémica pueden cursar con sarcopenia que puede acelerarse después de un estrés agudo inflamatorio. En algunos ancianos puede verse un bajo grado de respuesta inflamatoria sistémica o de resistencia a la insulina.

\section{Dos condiciones que se superponen}

A pesar de la clara distinción entre los conceptos de caquexia y sarcopenia, las investigaciones han demostrado que las dos condiciones tienen numerosas condiciones subyacentes. En la práctica clínica los factores que conducen a la pérdida de masa muscular en el contexto de las dos afecciones pueden ser indistinguibles en el paciente anciano. Una condición puede conducir a la otra y la contribución relativa a la pérdida de masa y fuerza musculares suele no ser clara. Esta observación sugiere también que el mismo tratamiento podría beneficiar ambas enfermedades. Si el mismo mecanismo fisiológico se centra en las dos, el objetivo terapéutico común es reducir la pérdida de masa y fuerza musculares con intervenciones apropiadas y combinadas. ${ }^{10}$

No hay que olvidar que con la caquexia coexisten otras enfermedades y factores de riesgo que contribuyen a la pérdida de masa muscular y complican la comprensión de los mecanismos involucrados. En primer lugar, las enfermedades agudas y las hospitalizaciones resultan en inactividad con periodos de reposo en cama que tienen un impacto dramático sobre la masa muscular. Contrario a la caquexia, durante este periodo no se produce degradación de proteínas y es la inhibición en la síntesis de proteínas la responsable de la pérdida de la masa. En personas mayores sanas, diez días de reposo en cama resulta en 0.027-0.047\% por hora la disminución en la síntesis de proteína muscular, pérdida 0.95 k de masa de las extremidades inferiores y una baja de $15.6 \%$ en la fuerza muscular. Este efecto negativo del reposo en cama sobre el músculo parece más importante en pacientes mayores que en jóvenes. Durante una afección aguda la caquexia a menudo se combina con el reposo en cama y el efecto de ambas condiciones puede resul tar en una pérdida aún más importante de masa y fuerza musculares. Incluso las intervenciones de nutrición y el entrenamiento de fuerza han demostrado ser rel evantes en los pacientes caquécticos para la prevención de la masa muscular. ${ }^{10}$

En segundo lugar, las citocinas proinflamatorias son incapaces de interferir en el control hormonal del metabolismo muscular. La IL-6 y el TNF- son causales de la resistencia a la insulina y al factor de crecimiento similar a la insulina (IGF-I), y de la reducción en los nivel es de testosterona y hormona luteinizante. Estos cambios hormonales observados durante el envejecimiento habitual, reducen la tasa de síntesis de proteínas musculares. 
En tercer lugar, la característica principal de la caquexia es la pérdida de peso relacionada en parte con anorexia y a reducción en la ingesta de alimentos. Los pobres resultados de la intervención nutricional sugieren que la anorexia es más una consecuencia que una causa de pérdida de peso. Varios factores como depresión, quimioterapia, dolor, dispepsia y anorexia del envejecimiento contribuyen a la reducción de la ingesta de alimentos en el contexto de enfermedad inflamatoria crónica. A demás, las citocinas producen un efecto anorexígeno en el hipotálamo.

Por último, los procesos inflamatorios considerados como característicos de la caquexia también deben verse como una superposición en la sarcopenia del envejecimiento. Esta relación ha demostrado ser un factor de riesgo para la disminución de la masa muscular y la aparición de la discapacidad funcional en las personas mayores. El aumento de la masa grasa durante el envejecimiento contribuye al aumento de citocinas proinflamatorias que actúan sinérgicamente para acel erar la pérdida de masa y fuerza musculares. Es paradójico que su presencia también aumenta la masa grasa y favorece su depósito dentro del músculo afectando la sensibilidad de la insulina por los músculos. Como resultado, los pacientes obesos tienen un mayor riesgo de sarcopenia y disminución en la funcionalidad.

\section{Aproximación terapéutica}

L a pérdida de masa muscular por caquexia o sarcopenia puede ser manejada a través de un enfoque múltiple que incluya ejercicio y suplementación nutricional. El fortalecimiento muscular es eficaz en la prevención de la pérdida de masa en varios modelos de atrofia mediada por desuso. El volumen y la intensidad de los ejercicios necesarios parecen ser mayores que los prescritos para la prevención de la atrofia, sin embargo muchos de los programas son adoptados de los planes conocidos para hipertrofia. ${ }^{41}$

Otro tipo de ejercicio como las vibraciones del cuerpo entero ha sido propuesto hace poco por su potencial para incrementar la fuerza en los miembros inferio- res. Su reciente popularidad se debe a los efectos combinados de los sistemas neuromusculares y neuroendocrinos. La evidencia indica que la vibración puede ser una intervención de ejercicio para reducir los resultados de los procesos del envejecimiento en las estructuras musculoesqueléticas. Sin embargo, son necesarios estudios futuros para entender el mecanismo neurofisiológico implicados en la activación muscular con la vibración, con el fin de poder prescribir programas seguros y eficaces. ${ }^{42}$

En cuanto a suplementación nutricional, la creatina ha sido sugerida como un agente profiláctico o terapéutico en enfermedades que afectan el tamaño y la función muscular. Ha mostrado efectos positivos sobre la cantidad de masa magra y la función muscular en pacientes con sarcopenia. Sin embargo, los estudios controlados con placebo han revelado efectos variables, con mejoras en algunos. ${ }^{43}$ O tra ayuda ergogénica sugerida es la L -carnitina, un aminoácido que facilita la transferencia de las cadenas largas de ácidos grasos desde el citoplasma a la mitocondria, donde son procesados por la oxidación para producir ATP. ${ }^{44}$ La L-carnitina ha demostrado mejorar el estado del paciente en diversas enfermedades incluyendo demencia senil, neuropatías metabólicas, infección por HIV, miopatías, cardiomiopatías y falla renal. ${ }^{45,46}$ La L-carnitina es esencial para la bioenergética del musculo esquelético. ${ }^{47} \mathrm{~A}$ lgunos estudios han demostrado que podría ser una opción terapéutica para la miopatía inducida por diversas etiologías. ${ }^{44}$ Se ha sugerido a la grelina como supl emento porque induce un estado energético con balance positivo a través de mecanismos dependientes o independientes de la hormona de crecimiento. El tratamiento con esta hormona puede usarse en un subgrupo de pacientes con caquexia cardiaca. ${ }^{48} \mathrm{~L}$ a grelina estimula la ingesta de al imentos, regula el sistema nervioso central para disminuir el flujo de los nervios simpáticos e inhibe la adiposidad de los cardiomicitos y las cél ulas endoteliales de manera independiente. A demás incrementa la ingesta y el peso corporal, puede optimizar la capacidad de ejercicio, mejora la función ventricular izquierda ${ }^{49}$ y disminuye los niveles plasmáticos de noradrenalina. ${ }^{50,51}$ 


\section{Conclusión}

U na definición clara del síndrome clínico de caquexia representaría un logro importante en la medicina clínica que permitiría su detección temprana, prevención y tratamiento oportunos y apropiados. $\mathrm{Ha}$ sido considerado por mucho tiempo como un evento tardío que complica la historia natural de muchas enfermedades crónicas. A demás la mala respuesta de la caquexia al tratamiento farmacológico y a las intervenciones nutricionales ha ll evado a la idea errónea de que el complejo metabólico de la caquexia es solo susceptible a cuidados pal iativos. Con el conocimiento progresivo de su bioquímica y mecanismos moleculares, el papel de la deficiencia nutricional en la caquexia es cada vez más claro. Esto pone en rel evancia la necesidad de intervenciones terapéuticas apropiadas para prevenir la evolución de la enfermedad. En este documento se presenta una definición y los criterios para el diagnóstico de sarcopenia relacionada con la edad, compuesta por la combinación de la pérdida de la masa muscular y la disminución de la fuerza. La condición responde fácil al entrenamiento de fuerza. A demás hay nuevos tratamientos nutricionales y farmacológicos que son opciones posibles. El grado de caquexia tiene correlación inversa con el tiempo de supervivencia de los pacientes y siempre implica un mal pronóstico. En los últimos años las enfermedades e incapacidades relacionadas con la edad han despertado un gran interés e importancia sanitaria. Deberían dedicarse más investigaciones al esclarecimiento de los factores y mediadores del proceso caquéctico tanto en caquexia como en sarcopenia, para desarrollar estrategias terapéuticas y preventivas de la pérdida de masa muscular tanto en la enfermedad como durante el envejecimiento sano. ${ }^{2}$

\section{Referencias}

1. Muscaritoli M, Anker SD, Argilés J, Aversa Z, Bauer JM, Biolo G, et al. Consensus definition of sarcopenia, cachexia and pre-cachexia: Joint document elaborated by Special Interest Groups (SIG) "cachexia-anorexia in chronic wasting diseases" and "nutrition in geriatrics". Clin Nutr. 2010 Apr; 29(2):154-9.

2. Argilés JM, Busquets S, López Soriano FJ, Figueras M. Fisiologia de la sarcopenia. Similitudes y diferencias con la caquexia neoplasica. Nutr Hosp. 2006;21(Supl 3):38-45

3. Schneider SM, Al-Jaouni R, Pivot X, Braulio VB, Rampal P, Hebuterne X Lack of adaptation to severe malnutrition in elderly patients. Clin Nutr. 2002;21(6):499-504.
4. Chandra RK. Nutrition, immunity and infection: present knowledge and future directions. Lancet. 1983; 1(8326 Pt 1):688-91.

5. Wallace JI, Schwartz RS, LaCroix AZ, Uhlmann RF, Pearlman RA. Involuntary weight loss in older outpatients: incidence and clinical significance. J Am Geriatr Soc. 1995;43(4):329-37.

6. Goodwin JS, Goodwin JM, Garry PJ. Assocication between nutritional status and cognition in a healthy elderly population. JAMA. 1983;249(21):2917-21.

7. Braun JV, Wykle MH, Cowling WR 3rd. Failure to thrive in older persons: a concept derived. Gerontologist. 1988;28(6):809-12.

8. Morley JE, Silver AJ. Anorexia in the enderly. Aging. 1998;9(1):9-16

9. Doehner W, Anker SD. Cardiac cachexia in early literature: a review of research prior to Medline. Int J Cardiol. 2002;85(1):7-14.

10. Rolland Y, Abellan van Kan G, Gillette-Guyonnet S, Vellas B. Cachexia versus sarcopenia. Curr Opin Clin Nutr Metab Care. 2011 Jan; 14(1):15-21.

11. Ize Lamache L. ¿Por qué no come el abuelo? Sarcopenia o anorexia. Nutr. Clín. 2003;6(1):53-7.

12. Burgos Peláez R. Global therapeutica approach to sarcopenia. Nutr Hosp. 2006;21(3):51-60.

13. Janssen I, Heymsfield SB, Ross R. Low relative skeletal muscle mass (sarcopenia) in older persons is associated with functional impairment and physical disability. J Am Geriatr Soc. 2002;50(5): 889-96.

14. Evans WJ. What is sarcopenia?. J Gerontol A Biol Sci Med Sci. 1995;50:5-8

15. Baumgartner RN, Koehler KM, Gallagher D, Romero L, Heymsfield SB, Ross $\mathrm{RR}$, et al. Epidemiology of sarcopenia among the elderly in New Mexico. Am J Epidemiol. 1998;147(8): 755-63.

16. Cruz-Jentoft AJ, Baeyens JP, Bauer JM, Boirie Y, Cederholm T, Landi F, et al Sarcopenia: European consensus on definition and diagnosis Report of the European Working Group on Sarcopenia in Older People. Age and Aging 2010; $39: 412-23$.

17. Rosenberg I. Summary comments. Am J Clin Nutr. 1989;50:1231-3

18. Roubenoff R. Origins and clinical relevance of sarcopenia. Can J Appl Physiol. 2001;26(1):78-89.

19. Serra Rexach JA. Consecuencias clínicas de la sarcopenia. Nutr Hosp. 2006;21(3):46-50.

20. Thompson DD. Envejecimiento y sarcopenia. Rev Metab Óseo y Min. 2011;9(4):140-1

21. Burgos Peláez R. Sarcopenia en ancianos. Endocrinol Nutr. 2006;52:335-45

22. Kamel HK. Sarcopenia and aging. Nutr Rev. 2003;61(157):167.

23. Becerra Urrego R, Galvis Rincón JC. Perfil hormonal del envejecimiento muscular. Repert Med Cir. 2011; 20(4): 217-24

24. Masanés Torán F, Navarro López M, Sacanella Meseguer E, López Soto A. ¿Qué es la sarcopenia?. Semin Fund Esp Reumatol. 2010;11(1):14-23.

25. Guralnik JM, Ferrucci L, Pieper CF, Leveille SG, Markides KS, Ostir GV, et al. Lower extremity function and subsequent disability: consistency across studies, predictive models, and value of gait speed alone compared with the short physical performance battery. J Gerontol A Biol Sci Med Sci 2000;55(4):M221-31.

26. Evans WJ, Morley JE, Argilés J, Bales C, Baracos V, Guttridge D, et al. Cachexia: a new definition. Clin Nutr. 2008;27(6):793-9.

27. Milke Garcia P, Rivera Flores R. Sindrome de anorexia-caquexia. Rev Gatroenterol Méx. 2010;75 (supl. 2): 205-7.

28. Springer J, von Haehling S, Anker SD. The need for a standardized definition for cachexia in chronic illness. Nat Clin Pract Endocrinol Metab. 2006;2:416-7.

29. Wolfe RR. Control of muscle protein breakdwon: effects of activity and nutritional states . Int J Sport Nutr Exerc Metab. 2001;11(supl.):S164-9.

30. Fearon KC, Voss AC, Hustead DS; Cancer Cachexia Study Group. Definition of cancer cachexia: effect of weight loss, reduced food intake and systemic inflammation on functional status and prognosis. Am J Clin Nutr. 2006;83:1345-50 
31. Anker SD, Coats AJ. Cachexia in heart failure is bad for you. Eur Heart J. 1998;19:191-3

32. Mulligan K, Tai VW, Schambelan M. Cross-sectional and longitudinal evaluation of body composition in men with HIV infection. J Acquir Immune Defic Syndr Hum Retrovirol. 1997;15(1):43-8.

33. Kalantar-Zadeh K, Kopple JD. Obesity paradox in patients on maintenance dialysis. Contrib Nephrol. 2006;151:57-69.

34. Martínez Roque V, Silencio Barrita, JL. Valoración del estado de nutrición en el paciente con cáncer. Cancerología. 2007;2:315-26.

35. Mézquita Ortiz JF, Arreola Viera MA, Bastarrachea Sosa RA. Fisiopatología de la caquexia. Med Int Mex. 2002;18(2):93-101.

36. Tisdale MJ. Cachexia in cancer patients. Nat Rev Cancer. 2002;2:862-71.

37. Ockenga J, Valentini L. Review article: anorexia and cachexia in gastrointestinal cancer. Aliment Pharmacol Ther. 2005;22:583-94.

38. Strasser F. Pathophysiology of the anorexia/cachexia syndrome. In: Doyle D, Hanks G, Cherny NI, editors. Oxford textbook of palliative medicine. 3rd ed. New York: Oxford University; 2004. p. 520-33

39. Davis MP, Dickerson D. Cachexia and Anorexia: cancer's covert killer. Support Care Cancer. 2000;8:180-7.

40. Cereceda G Luis. Mecanismos de la Caquexia. Rev Med Clin Condes. 2007;18(4):349-55.

41. Kotler DP. Cachexia. Ann Intern Med. 2000;133:622-34.

42. Glover EI, Phillips SM. Resistance exercise and appropriate nutrition to counteract muscle wasting and promote muscle hypertrophy. Curr Opin Clin Nutr Metab Care. 2010;13:630-4.
43. Cardinale M, Wakeling J. Whole body vibration exercise: are vibrations good for you?. Br J Sports Med. 2005 Apr 25;39:585-9.

44. Sakkas GK, Schambelan M, Mulligan K. Can the use of creatine supplementation attenuate muscle loss in cachexia and wasting?. Curr Opin Clin Nutr Metab Care. 2009;12:623-7.

45. Silvério R, Laviano A, Rossi Fanelli F, Seelaender M. L-carnitine and cancer cachexia: Clinical and experimental aspects. J Cachexia Sarcopenia Muscle. 2011 Jan 26;2:37-44.

46. Evangeliou A, Vlassopoulos D. Carnitine metabolism and deficit - when supplementation is necessary?. Curr Pharm Biotechnol. 2003;4:211-9.

47. Laviano A, Meguid MM, Guijarro A, Muscaritoli M, Cascino A, Preziosa I, et al. Antimyopathic effects of carnitine and nicotine. Curr Opin Clin Nutr Metab Care. 2006;9:442-8.

48. Brass EP. Supplemental carnitine and exercise. Am J Clin Nutr. 2000;72:618S$23 \mathrm{~S}$

49. Akashi YJ, Springer J, Anker SD. Cachexia in chronic heart failure: prognostic implications and novel therapeutic approaches. Curr Heart Fail Rep. 2005;2:198203.

50. Nagaya N, Moriya J, Yasumura Y, Uematsu M, Ono F, Shimizu W. Effects of ghrelin administration on left ventricular function, exercise capacity, and muscle wasting in patients with chronic heart failure. Circulation. 2004;110:3674-9.

51. Akamizu T, Kangawa K. Ghrelin for cachexia. J Cachexia Sarcopenia Muscle. 2010;1:169-76. 